\title{
Mentoring zur klinischen und akademischen Karriereförderung junger Ärztinnen und Ärzte
}

\author{
B. Buddeberg-Fischer ${ }^{a}$, B. Beck-Schimmer ${ }^{b}$, R. Hornung', C. Dietz ${ }^{a}$, G. Mattanza ${ }^{a}$, R. Klaghofer ${ }^{a}$
}

UniversitätsSpital Zürich:

a Psychosoziale Medizin

b Institut für Anästhesiologie

c Klinik für Gynäkologie

* Das Programm wird gefördert im Rahmen des Bundesprogramms Chancengleichheit Modul 2 Mentoring (M04/39).

1 Healy CC, Welchert AJ. Mentoring relations: a definition to advance research and practice. Am Educ Res J 1990;19:17-21.

2 Bhagia J, Tinsley JA. The mentoring partnership. Mayo Clinic Proceedings 2000;75:535-7.

3 Buddeberg-Fischer B, Vetsch E, Mattanza G. Career support in medicine: experiences with a mentoring program for junior physicians at a university hospital Psycho-Social-Medicine 2004. www.egms.de/en/journals/psm/ 2004-1/psm000004.shtml

Korrespondenz:

Prof. Dr. med.

Barbara Buddeberg-Fischer

Psychosoziale Medizin

UniversitätsSpital Zürich

Haldenbachstrasse 18

CH-8091 Zürich

Tel. 0442555127

Fax 0442554384

E-Mail: barbara.buddeberg@usz.ch

\section{Zusammenfassung}

Mentoring als Instrument zur Nachwuchsförderung von Ärztinnen und Ärzten wird in der Medizin in deutschsprachigen Ländern bisher kaum eingesetzt. Das dargestellte Mentoringprogramm* ist Bestandteil von Fördermassnahmen zur «Gleichberechtigten Nachwuchsförderung von Ärztinnen und Ärzten» am UniversitätsSpital Zürich. Im folgenden wird dieses neuentwickelte Mentoringmodell vorgestellt: Gruppen von Ärztinnen und Ärzten (Peers/Mentees) werden von Mentorinnen/Mentoren und der Programmleiterin im Mentoringprozess beraten und in der Umsetzung von Karriereschritten unterstützt. Es erfolgt eine Begleitevaluation mittels quantitativer und qualitativer Methoden. Als Motivation zur Teilnahme am Mentoringprogramm wurden folgende Gründe genannt: Information über Karrieremöglichkeiten, Erfahrungsaustausch innerhalb der Gruppe, Unterstützung beim Entwickeln von Karriereplänen und bei der Umsetzung von Karriereschritten, Networking in der «scientific community». Messbare Karriereschritte waren Aufstieg in Kaderpositionen, Zusatzqualifikationen, speditivere Weiterbildung, gesteigerte Forschungs- und Publikationstätigkeit sowie Stipendien für Forschungsaufenthalte im Ausland. Als Wirkfaktoren des Mentorings bezeichneten die Mentees u.a. die Konkretisierung eigener Karriereziele, den Erfahrungsaustausch innerhalb der Peergruppen, die Vertraulichkeit der Gespräche, die Unterstützung und Motivierung durch die Mentorinnen/Mentoren sowie die Strukturierung des Gruppenprozesses durch die Programmleitung. Mentoringprogramme sind eine effiziente Massnahme zur Nachwuchsförderung und sollten von Spitalund Klinikleitungen nachhaltig unterstützt werden.

\section{Mentoring und Mentoringprogramme}

In den letzten Jahren ist in der Medizin die Notwendigkeit erkannt worden, junge Ärztinnen und Ärzte frühzeitig und zielorientiert in ihrer Karriere zu beraten. Mentoring stellt eine struk- turierte Förderung einzelner Personen einer Berufsgruppe dar, mit dem Ziel einer optimalen Unterstützung ihrer Berufslaufbahn sowie ihrer persönlichen Entwicklung. Healy und Welchert [1] definieren die Mentoringbeziehung im beruflichen Kontext: «Mentoring is a dynamic reciprocal relationship in a work environment between two individuals where, often but not always, one is an advanced career incumbent and the other is a less experienced person. The relationship is aimed at fostering the development of the less experienced person.» Mentoring ist im Unterschied zu Coaching oder Counselling eine unentgeltliche Fördermassnahme. Ein/e Mentor/in unterscheidet sich auch von einem/einer Supervisor/in, Lehrer/in oder einem Rollenmodell [2]. Diese Beziehungsformen sind hierarchischer und «top down» strukturiert. Das Wesentliche des Mentoringprozesses ist die Reziprozität der Beziehung, beide - Mentor/in und Mentees - profitieren von dem Förderinstrument. Im Mentoringprozess lassen sich fünf Phasen unterscheiden: Informieren über Karrieremöglichkeiten («Forming»), Entwickeln von Karriereplänen («Storming»), Fokussieren auf Karriereziele («Norming»), Umsetzen von Karriereschritten («Performing») und Evaluieren von Karriereerfolgen («Finalizing») [3].

Zur Karriereförderung gibt es unterschiedliche Mentoringmodelle: Das dyadische Mentor/inMentee-Modell, bei dem eine erfahrene Fachperson (Mentor/in) z.B. einzelne Assistierende (Mentees) hinsichtlich ihrer Berufslaufbahn berät und fördert. Die meisten Mentoringbeziehungen basieren auf diesem One-to-one-Modell. Daneben gibt es Peermentoring, bei dem sich Assistierende (Peers) gegenseitig in ihren Karrierebestrebungen unterstützen. Erfahrungen mit beiden Modellen haben gezeigt, dass in der Medizin, speziell in grossen und komplexen Systemen wie Universitätskliniken, eine Integration dieser beiden Modellansätze vorteilhaft ist: Dies bedeutet, dass sich eine kleine Gruppe von Ärztinnen und Ärzten, welche eine vertrauensvolle Beziehung zueinander haben, gemeinsam eine Fachperson als Mentorin bzw. Mentor wählen. Da viele Mentorinnen/Mentoren vor allem zu Beginn ihrer Mentorenschaft wenig Erfahrung 
im Führen von Gruppen haben und oft selbst noch im Aufbau ihrer Karriere sind, ist eine zielund prozessorientierte Begleitung und Beratung der Gruppen durch eine externe Fachperson hilfreich. Diese Fachperson übernimmt die Koordination der Mentoringaktivitäten und die Funktion der Programmleitung und -evaluation. Vor allem in Konfliktsituationen zwischen Klinikdirektion und Assistierenden können Mentorinnen/Mentoren ohne die allparteiliche Unterstützung einer externen Fachperson in einen Loyalitätskonflikt zwischen den verschiedenen Hierarchieebenen geraten.

\section{Mentoringprogramm am Universitäts- Spital Zürich}

\section{Programmdesign}

Die Spitalleitung des UniversitätsSpitals Zürich (USZ) erteilte den Autorinnen und Autoren im Jahr 2001 den Auftrag, ein Mentoringprogramm für Assistierende beiderlei Geschlechts auszuarbeiten. Nach einer zwölfmonatigen Pilotphase im Jahr 2002, über welche an anderer Stelle berichtet wurde [3], ist das Programm seit 2003 als fester Bestandteil der Nachwuchsförderung am USZ etabliert. Die Struktur dieses Mentoringprogramms ist in Abbildung 1 dargestellt.

Das Mentoringprogramm wird in folgender Siegrist J, Starke D, Chandola T, Godin I, Marmot M, Niedhammer et al. The measurement of effortreward imbalance at work: European comparisons. Soc Sci Med 2004;58:1483-9.
Weise bekanntgemacht: Alle neueintretenden Ärztinnen und Ärzte erhalten einen Flyer mit Informationen zum Mentoringprogramm. Zusätz-

\section{Abbildung 1}

Organisationsstruktur des Mentoringprogramms.

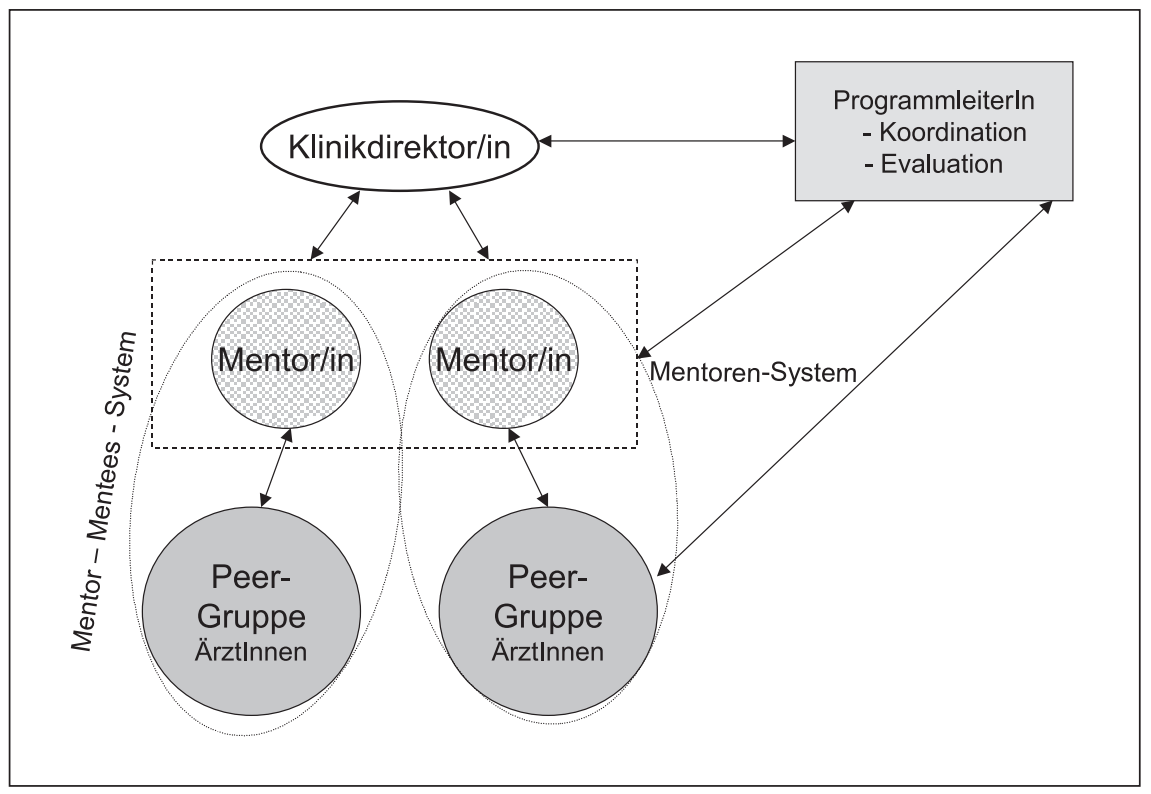

lich gibt es im Intranet des USZ einen Link zum Mentoringprogramm. Schliesslich findet einmal jährlich eine spezielle Informationsveranstaltung statt. Die meisten Mentees sind in Gruppen organisiert, es haben sich aber auch One-to-oneMentoringbeziehungen entwickelt.

\section{Teilnehmende Mentees}

50 Mentees (38 Frauen und 12 Männer) nahmen von Januar 2003 bis Juni 2005 am Programm teil. Sie sind durchschnittlich 34 Jahre alt, haben im Mittel vor $6 \frac{1}{2}$ Jahren das Medizinische Staatsexamen abgelegt und arbeiten seit durchschnittlich fünf Jahren in ihrem angestrebten Fachgebiet. Ärztinnen und Ärzte aus folgenden Fachdisziplinen sind vertreten: Anästhesiologie, Chirurgie, Dermatologie, Frauenheilkunde, Innere Medizin, Klinische Pharmakologie und Ophthalmologie. Bei Eintritt in das Programm hatten die Mentees Assistenzarztstellen inne; einige Mentees sind im Verlauf des Programms zu Oberärztinnen/-ärzten befördert worden. Nur drei Ärztinnen und zwei Ärzte haben bereits eigene Kinder. Die durchschnittliche Teilnahmedauer am Mentoringprogramm beträgt 1 1 $\frac{1}{2}$ Jahre (Streubreite 6-32 Monate). Die Hälfte der Mentees $(n=25)$ streben eine klinische, zehn eine akademische Laufbahn an, sechs wollen später in einer Praxis arbeiten, ein/e Mentee hat eine Industrielaufbahn eingeschlagen und acht sind noch unentschieden.

\section{Mentoren}

Die Mentoren (3 Frauen und 2 Männer) sind Leitende bzw. Oberärztinnen/-ärzte, die selbst eine akademische Karriere durchlaufen haben oder klinische Kaderpositionen einnehmen.

\section{Begleitevaluation - Instrumente}

Das Mentoringprogramm wird mittels Fragebogen evaluiert. Im vorliegenden Artikel werden Ergebnisse der Initialbefragung, d.h. bei Eintritt ins Programm, berichtet. Folgende validierte Instrumente zur Charakterisierung der teilnehmenden Mentees kamen zur Anwendung:

- Effort-reward Imbalance Questionnaire ERI (zwei fünfstufige Skalen: «effort» misst das Mass der beruflichen Verausgabungsneigung und «reward» die erfahrene berufliche Gratifikation; die vierstufige Skala «overcommitment» erfasst die Tendenz zum beruflichen Überengagement) [4]. Das Verhältnis von effort und reward (ERI) sollte ausgeglichen, d.h. der Quotient $<1$ sein. Werte $>1$ deuten auf ein ungünstiges Verhältnis zwischen beruflichem Engagement und erhaltener Anerkennung hin. 
- Fragebogen zur beruflichen Motivation (drei siebenstufige Skalen: «intrinsische» und «extrinsische Motivation» sowie «extraprofessionale Belange») [5].

- Personal Attributes Questionnaire GE-PAQ (zwei sechsstufige Skalen: «Instrumentalität» misst die Handlungsorientierung und Entscheidungsfreudigkeit, «Expressivität» beschreibt die Beziehungsorientierung und Hilfsbereitschaft) [6].

- Sense of Coherence Scale (siebenstufige Kurzform SOC-13) [7] misst das Kohärenzgefühl einer Person, d.h. die Fähigkeit, Stress zu bewältigen und Belastungen als Herausforderungen zu erleben.

Ausserdem nannten die Mentees Motive/Gründe für die Teilnahme am Programm und welche beruflichen Ziele sie für die nächsten 1 bis 2 Jahre anstreben wollen.

Als Referenzstichprobe für die im folgenden berichteten Ergebnisse dient eine Stichprobe junger Schweizer Ärztinnen und Ärzte ( $\mathrm{n}=518)$, die an einer Longitudinalstudie zu Determinanten der Karriereentwicklung teilnehmen [8, 9], sich ebenfalls in fachärztlicher Weiterbildung befinden und ungefähr gleich alt sind.

\section{Statistische Auswertung}

Sämtliche Auswertungen wurden mit dem Programm SPSS für Windows, Version 12.0, durchgeführt. Deskriptive Statistiken sind mit Häufigkeiten und Prozentwerten bzw. mit Mittelwerten und Standardabweichungen angegeben. Der Vergleich von Skalenmittelwerten mit Referenzwerten erfolgte mittels One-sample-t-Test.

\section{Ergebnisse}

\section{Charakterisierung der Mentees}

Das Mentoringprogramm steht grundsätzlich allen Ärztinnen und Ärzten am UniversitätsSpital Zürich offen. Uns interessierte, wie die teilnehmenden Mentees ihre Arbeitssituation und Karrieremotivation einstufen, welche Persönlichkeitsmerkmale sie aufweisen und wie sie ihre Lebenszufriedenheit und ihre psychische Befindlichkeit einschätzen. Die Angaben der Mentees wurden mit Daten einer Referenzstichprobe von altersentsprechenden Schweizer Ärztinnen und Ärzten in fachärztlicher Weiterbildung [9] verglichen (Tab. 1). Die Mentees zeigen eine ähnlich hohe berufliche Verausgabungsneigung wie die Teilnehmenden der Longitudinalstudie zur beruflichen Entwicklung junger Ärztinnen und Ärzte, sie erhalten aber signifikant weniger berufliche Gratifikation für ihr Engagement, d.h., das Verhältnis von beruflichem Einsatz und erhaltener Anerkennung ist ungünstiger. Dabei stufen die Mentees ihre intrinsische (Freude und Interesse an der beruflichen Tätigkeit selbst) und extrinsische Motivation (Interesse an beruflichem Aufstieg) signifikant höher ein und messen dementsprechend den extraprofessionalen Belangen (familiären und Freizeitinteressen) eine weniger
5 Abele AE. Karriereorientierungen angehender Akademikerinnen und Akademiker. Bielefeld: Kleine; 1994.

6 Alfermann D. Androgynie. In: Reigber D (Hrsg.). Frauen-Welten. Düsseldorf: Econ; 1993. S. 147-200.

7 Antonovsky A. Unraveling the Mystery of Health. How People Manage Stress and Stay Well. San Francisco: Jossey-Bass; 1987.

8 Buddeberg-Fischer B, Klaghofer R, Abel T, Buddeberg C. The influence of gender and personality traits on the career planning of medical students. Swiss Med Wkly 2003; 133:535-40.

9 Buddeberg-Fischer B, Klaghofer R, Abel T, Buddeberg C. Junior physicians' workplace experiences in clinical fields in German-speaking Switzerland. Swiss Med Wkly 2005;135(1-2):19-26.

\section{Tabelle 1}

Arbeitssituation, Karrieremotivation und Persönlichkeitsmerkmale der Mentees im Vergleich zu einer Referenzstichprobe junger Ärztinnen/Ärzte. Mittelwerte und Standardabweichungen (in Klammern).

\begin{tabular}{|c|c|c|c|}
\hline Skala & Mentees $(n=50)$ & Referenzstichprobe $(n=518)$ & $\mathbf{P}$ \\
\hline \multicolumn{4}{|l|}{$E R I$} \\
\hline Effort & $15,72(3,42)$ & $15,13(3,94)$ & 0,23 \\
\hline Reward & $40,20(5,88)$ & $43,57(6,28)$ & $<0,001$ \\
\hline ERI & $0,88(0,22)$ & $0,80(0,31)$ & 0,02 \\
\hline Overcommitment & $2,24(0,59)$ & $2,25(0,58)$ & 0,91 \\
\hline \multicolumn{4}{|l|}{ MOT } \\
\hline intrinsisch & $6,32(0,36)$ & $6,08(0,57)$ & $<0,001$ \\
\hline extrinsisch & $4,37(0,70)$ & $4,05(0,90)$ & $<0,01$ \\
\hline extraprofessionell & $3,95(1,08)$ & $4,30(1,05)$ & 0,03 \\
\hline \multicolumn{4}{|l|}{ PAQ } \\
\hline Instrumentalität & $4,11(0,69)$ & $4,19(0,69)$ & 0,40 \\
\hline Expressivität & $5,04(0,54)$ & $4,88(0,56)$ & 0,04 \\
\hline SOC & $5,18(0,78)$ & $5,08(0,86)$ & 0,36 \\
\hline
\end{tabular}


grosse Bedeutung zu. Sie schätzen ihre Expressivität höher ein als die Vergleichsstichprobe. Bezüglich Instrumentalität und Kohärenzgefühl bestehen keine Unterschiede zwischen den Mentees und den Ärztinnen und Ärzten der Longitudinalstudie. Beide Gruppen geben im Vergleich zur Normalbevölkerung relativ hohe Werte für das Kohärenzgefühl an.

\section{Erwartungen an das Mentoringprogramm}

Bei Eintritt in das Mentoringprogramm formulierten die Mentees, welche Erwartungen sie mit der Teilnahme am Programm verknüpften (Tab. 2). Ein Drittel der Nennungen entfiel auf Themen, die der ersten Phase des Mentoringprozesses, dem «Forming», zugeordnet werden können: Erfahrungsaustausch und Information über Karrieremöglichkeiten. Knapp ein Viertel der Nennungen betraf das Entwickeln von Karriereplänen (Phase des «Storming»). Ein Fünftel umfasste Erwartungen hinsichtlich der Konkretisierung von Karrierezielen («Norming»), ein weiteres Fünftel konnte dem «Performing» (Unterstützung bei der Umsetzung von Karriereschritten) zugeordnet werden.

\section{Angestrebte berufliche Ziele}

Bei der Planung der beruflichen Karriere sind Entscheidungen auf verschiedenen Ebenen zu fällen, z.B., ob man eine wissenschaftliche oder eine praxisorientierte klinische Laufbahn anstrebt. Daneben ist es sinnvoll, sich jeweils für einen überschaubaren Zeitraum kleinere Karriereziele zu stecken und diese dann konkret zu pla- nen und umzusetzen. Umschriebene Karriereziele können besser evaluiert werden und tragen bei Erreichen des Ziels zur Motivation für die weitere Karriereplanung bei. Die Mentees des Programms wurden deshalb aufgefordert, für die nächsten 1-2 Jahre ihre angestrebten Karriereziele $\mathrm{zu}$ formulieren (Tab. 3). Da die meisten Mentees noch in der fachärztlichen Weiterbildung standen, entfiel über die Hälfte der Nennungen auf die Optimierung der Weiterbildung bzw. eine erfolgreich bestandene Facharztprüfung. Ein Viertel der Nennungen betraf wissenschaftliche Karriereziele wie die Durchführung von Forschungsprojekten, das Schreiben von Publikationen, Auslandsaufenthalte und Integration in Forschungsnetzwerke. Andere Ziele fokussierten auf die Zeit nach Abschluss der Weiterbildung wie z.B. den Erwerb von Oberarztkompetenzen oder Subspezialisierungen.

\section{Diskussion}

Die Erfahrungen und Ergebnisse des Mentoringprogramms zeigen, dass es sich bei den Mentees um beruflich hochmotivierte junge Ärztinnen und Ärzte handelt, die zu einem grossen beruflichen Engagement auch auf Kosten ihres Privatlebens bereit sind. Sie haben die Fähigkeit, anstehende Belastungen als persönliche Herausforderung zu sehen und sind überzeugt, diese erfolgreich meistern zu können. Neben ihrer Karriereorientierung sind sie beziehungsorientierte, d.h. auch teamfähige Personen. Aus den

\section{Tabelle 2}

Häufigkeitsverteilung der 226 Nennungen der Erwartungen an das Mentoringprogramm (MP) der 50 Mentees (gruppiert nach Kategorien) (in Klammern \%).

\begin{tabular}{|c|c|c|c|}
\hline Erwartungen an das MP & $\begin{array}{l}\text { Nennungen } \\
\text { Total } \mathrm{N}\end{array}$ & $\begin{array}{l}\text { Nennungen } \\
\text { von Frauen } \mathbf{n}\end{array}$ & $\begin{array}{l}\text { Nennungen } \\
\text { von Männern } n\end{array}$ \\
\hline \multicolumn{4}{|l|}{ «Forming» } \\
\hline Erfahrungsaustausch & $42(18,6)$ & $30(13,3)$ & $12(5,3)$ \\
\hline Informationen zu Karrieremöglichkeiten & $39(17,3)$ & $30(13,3)$ & $9(4,0)$ \\
\hline \multicolumn{4}{|l|}{ «Storming» } \\
\hline Entwickeln von Karriereplänen & $50(22,1)$ & $38(16,8)$ & $12(5,3)$ \\
\hline \multicolumn{4}{|l|}{ «Norming» } \\
\hline Konkretisierung von Karrierezielen & $40(17,7)$ & $30(13,3)$ & $10(4,4)$ \\
\hline \multicolumn{4}{|l|}{ «Performing» } \\
\hline $\begin{array}{l}\text { Unterstützung bei der Umsetzung } \\
\text { von Karriereschritten }\end{array}$ & $44(19,5)$ & $33(14,6)$ & $11(4,8)$ \\
\hline Andere Gründe & $11(4,8)$ & $9(4,0)$ & $2(0,9)$ \\
\hline Total Nennungen & $226(100)$ & $170(75,2)$ & $56(24,8)$ \\
\hline
\end{tabular}


Tabelle 3

Häufigkeitsverteilung der 148 Nennungen der beruflichen Ziele der 50 Mentees für die nächsten 1-2 Jahre (in Klammern \%).

\begin{tabular}{|c|c|c|c|}
\hline Berufliche Ziele & $\begin{array}{l}\text { Nennungen } \\
\text { Total } N\end{array}$ & $\begin{array}{l}\text { Nennungen } \\
\text { von Frauen } \mathbf{n}\end{array}$ & $\begin{array}{l}\text { Nennungen } \\
\text { von Männern } n\end{array}$ \\
\hline Optimierung der Weiterbildung & $64(43,2)$ & $54(36,5)$ & $10(6,8)$ \\
\hline Konkretisierung der Karriereplanung & $19(12,8)$ & $14(9,5)$ & $5(3,4)$ \\
\hline Facharztprüfung & $18(12,2)$ & $13(8,8)$ & $5(3,4)$ \\
\hline $\begin{array}{l}\text { Planung und Durchführung von Forschungs- } \\
\text { projekten / Integration in Forschungsnetzwerke }\end{array}$ & $17(11,5)$ & $14(9,5)$ & $3(2,0)$ \\
\hline Schreiben von Publikationen & $12(8,1)$ & $7(4,7)$ & $5(3,4)$ \\
\hline Forschungsaufenthalt im Ausland & $5(3,4)$ & $1(0,7)$ & $4(2,7)$ \\
\hline Work-Life-Balance & $4(2,7)$ & $2(1,3)$ & $2(1,3)$ \\
\hline Erwerb von Oberarztkompetenzen & $3(2,0)$ & $2(1,3)$ & $1(0,7)$ \\
\hline $\begin{array}{l}\text { Subspezialisierung / Erwerb eines Fähigkeits- } \\
\text { ausweises }\end{array}$ & $2(1,3)$ & $2(1,3)$ & $0(0)$ \\
\hline Abschluss der Dissertation & $1(0,7)$ & $1(0,7)$ & $0(0)$ \\
\hline Diverses & $3(2,0)$ & $2(1,3)$ & $1(0,7)$ \\
\hline Total der Nennungen & $148(100)$ & $112(75,7)$ & $36(24,3)$ \\
\hline
\end{tabular}

Ergebnissen der Fragebogen sowie aus den Gesprächen in den Mentoringgruppen wurde jedoch deutlich, dass die jungen Kolleginnen und Kollegen für ihren hohen beruflichen Einsatz ihrer Meinung nach innerhalb der Institution und von ihren Vorgesetzten zu wenig Anerkennung und Unterstützung erhalten. Fehlende Anerkennung für geleistete Arbeit ist nach unseren Erfahrungen ein entscheidender Faktor, anspruchsvolle Karriereziele nicht weiter zu verfolgen. Aufgabe der Mentorinnen/Mentoren und der Programmleiterin ist es, den jungen Kolleginnen und Kollegen Ideen für die Umsetzung ihrer einzelnen Karriereschritte zu geben, sie fachlich zu beraten und aktiv in berufliche Netzwerke zu integrieren; wichtig ist aber auch, Wege zur Bewältigung von Schwierigkeiten aufzuzeigen, Stagnation und Rückschläge in der beruflichen Laufbahn zu relativieren und die Mentees zu motivieren, anvisierte Karriereziele nicht aufzugeben.

Die von den Mentees genannten Erwartungen an das Programm können den aus der Literatur bekannten Phasen im Mentoringprozess zugeordnet werden [3]. Dies zeigt, dass die Mentees realistische und umsetzungsadäquate Vorstellungen von Mentoring haben. Viele der Erwartungen und Ziele konnten im Verlauf der gemeinsamen Arbeit von Mentees, Mentorinnen/ Mentoren und Programmleitung bisher schon umgesetzt werden. Da die Dauer von Mentoringgruppen unterschiedlich ist und einzelne erst seit einigen Monaten im geschilderten Programm arbeiten, wurde von einer systematischen Erfolgsevaluation des Programms zum jetzigen
Zeitpunkt abgesehen. Von den 50 Teilnehmenden konnten bisher folgende Karriereschritte gemacht werden: 9 Mentees haben Oberarztpositionen übernommen, 6 Mentees sind auf dem Weg zu einer Habilitation entscheidende Schritte vorangekommen und 4 Mentees haben einen Forschungsaufenthalt im Ausland angetreten.

Mentoring ist für alle Beteiligten ein Gewinn. Einige Zitate der am Programm teilnehmenden Mentees und Mentorinnen/Mentoren veranschaulichen am besten, in welcher Weise sowohl die einzelnen im Mentoringprozess involvierten Personen als auch die Institution, in der das Programm etabliert ist, davon profitieren (siehe Kasten).

\section{Empfehlungen zur Implementierung von Mentoringprogrammen}

Aus den Erfahrungen des seit 2002 am USZ laufenden Mentoringprogramms lassen sich folgende Empfehlungen hinsichtlich günstiger Voraussetzungen und Rahmenbedingungen zur Implementierung von Mentoringprogrammen in einem Universitätsspital oder grossen Klinikum ableiten (Abb. 2):

- Institutionelle Rahmenbedingungen: «Commitment», d.h. die Spitalleitung erkennt Mentoring als wesentlichen Bestandteil ihrer Unternehmensstrategie und betrachtet Mentoring als Kriterium für Personalentwicklung und Qualitätsmanagement. Dies beinhaltet die Bereitstellung von Ressourcen wie Räume für die Gruppenmeetings, finanzielle Mittel für die Programmleitung und nicht zuletzt die Möglichkeit, solche Projekte innerhalb 
und ausserhalb des Klinikums bekanntzumachen. Auch die Klinikdirektorinnen/-direktoren sind für das Gelingen von Mentoringaktivitäten verantwortlich. Nur wenn sie sich in den Prozess involvieren lassen, kann das Ziel eines Mentoringprogramms - die optimale Förderung von jungen Kolleginnen und Kollegen in ihrer beruflichen und persönlichen Laufbahn - erreicht werden.

- Der/die Programmleiter/in verfügt günstigerweise über Erfahrungen in der systemischen Beratung und in der Leitung von Gruppen. Er/sie sollte Gruppenprozesse erkennen und steuern, die Auswirkungen der Gruppenarbeit auf die verschiedenen Ebenen des Kliniksystems abschätzen, positive und negative Rückkoppelungsprozesse beachten und sich allparteilich gegenüber den verschiedenen Subsystemen verhalten können. Ferner sollte er/sie die Fähigkeit haben, die am Mentoringprozess Beteiligten immer wieder neu zu motivieren. Auch Kenntnisse der formalen Rahmenbedingungen und informellen Regeln für eine Karriere in der Medizin sind wichtig. Während der/die Mentor/in die Mentees in fachlicher Hinsicht berät, beachtet der/die Programmleiter/in den Gruppenprozess und den systemischen Aspekt der Arbeit.

- Eine wichtige Voraussetzung eines Mentoringprogramms ist die «Freiwilligkeit», d.h.,

Abbildung 2

Günstige Voraussetzungen und Rahmenbedingungen für Mentoringprogramme.

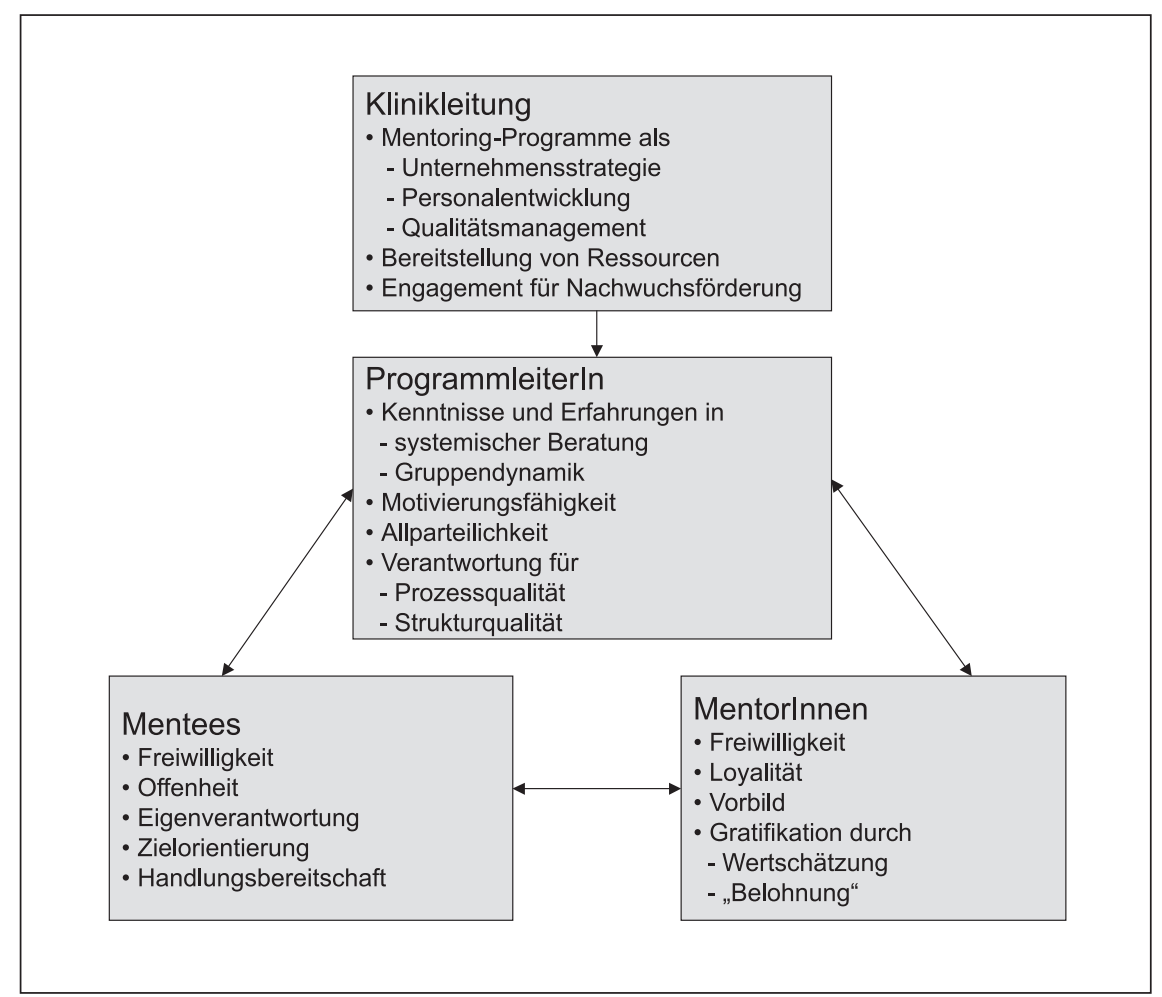

die Teilnahme sollte sowohl für Mentorinnen/Mentoren als auch Mentees freiwillig sein. Die Mentees sollten ihren/ihre Mentor/in frei wählen können, die Mentorinnen/Mentoren sich aber auch frei fühlen, eine Mentorenschaft zu übernehmen oder abzulehnen.

Erfahrungen von Mentorinnen/Mentoren

- «Als Mentor von jüngeren Kolleginnen und Kollegen ausgewählt zu werden, werte ich als Vertrauensbeweis. Es ist auch ein Feedback, wie ich im Klinikteam wahrgenommen werde.»

_ «Mentoring ist für mich eine neue Erfahrung im Umgang mit Assistierenden. Als Leitende Ärztin ist mir die stop down〉Beziehung viel vertrauter als das «bottom up in der Förderung und Beratung von jüngeren Kolleginnen und Kollegen.»

- «Ich bin überrascht, wie wenig konkrete Vorstellungen Assistierende $\mathrm{zu}$ ihrer beruflichen Zukunft haben. Teilweise komme ich mir wie ein Zugpferd vor, vor allem wenn es darum geht, die Begeisterung für die Forschung zu wecken und aufrechtzuerhalten.»

- «Während der Bereitschaftsdienste hat man als Oberarzt Gelegenheit, jüngere Kolleginnen und Kollegen persönlich und beruflich $\mathrm{zu}$ beraten. Es ist aber schwierig, abzuschätzen, wie weit man sich in die persönliche Sphäre vorwagen darf. Der strukturierte Rahmen eines Mentoringprogramms schafft eine gewisse Distanz, um auch persönliche Ratschläge zu geben. Die gemeinsame Führung der Gruppe durch den Fachmentor und die Programmleiterin gewährleistet eine gewisse Objektivität in der Beratung und neutralisiert subjektive Gefühle von Sympathie und Antipathie gegenüber einzelnen Personen.»

- «Durch die Beratung jüngerer Kolleginnen und Kollegen werde ich immer wieder dazu angeregt, auch meine eigene Karriere zu reflektieren.»

- «Das Mentoringprogramm gibt den Mentorinnen/Mentoren als Mitglieder des oberen Führungskaders die Möglichkeit, institutionalisierte Impulse in Richtung einer modernen Führungskultur auszulösen (flachere Hierarchien, transparentere Kommunikation und Mitarbeiterbeurteilungen).» 
- Die Mentorinnen/Mentoren sollten für die Mentees nicht in der Rolle der direkt vorgesetzten Oberärztinnen/-ärzte stehen und sich gegenüber den Mentees loyal und gegenüber der Klinikdirektion verschwiegen zeigen. Aufgrund ihres fachlichen Wissens,

\section{Erfahrungen von Mentees}

- «Die Fragebogenerhebung bei Eintritt in die Mentoringgruppe regte dazu an, einmal konkret über kurz-, mittel- und langfristige Karriereziele nachzudenken und diese auch schriftlich festzuhalten. Für mich war dies ein wichtiger erster Schritt in meiner Karriereplanung.»

_ «Positiv erlebte ich die Solidarität in der Gruppe: Wir motivierten uns gegenseitig und entwickelten auf der Basis der unterschiedlichen Erfahrungen miteinander Strategien für die Karrierewege der einzelnen Mitglieder. Ganz wichtig war die Vertraulichkeit der Gespräche, es wurden keine Informationen aus der Gruppe nach aussen getragen.»

- «Als hilfreich erlebte ich auch die Anwesenheit der Programmleiterin. Sie strukturierte unsere Treffen und konnte uns Mentees von einem objektiven Standpunkt aus und unbeeinflusst von der jeweiligen Situation in der Klinik beraten.»

- «Es ist für mich das erste Mal, dass ich mit jemandem vertraulich über meine Karriere reden konnte und konkrete Empfehlungen erhalten habe. Bisher haben mich nur Vorgesetzte beraten, die von meiner Forschungstätigkeit auch für ihre eigene Karriere profitieren wollten.»

- «Besonders gut hat mir gefallen, dass die Programmleiterin auch eine Frau ist und einen beachtenswerten Karriereweg hinter sich hat. Wichtig finde ich, dass man von jemand beraten wird, der das System einer universitären Institution kennt. Es ist oft schwer, selbst Entscheidungen zu treffen, wenn man einem Problem zu nahe steht. Man sieht die Situation nicht mehr objektiv genug. Mir haben die unabhängige Blickweise und Einschätzung von aussen viel geholfen.»

- «Dass viele Nachwuchskräfte ähnliche Schwierigkeiten in ihrer Karriere erleben, tröstet mich wenig. Das macht mich kampfeslustig, weil ich das so nicht akzeptieren mag. Ich denke, da muss sich noch etwas im System ändern.» ihrer Stellung innerhalb der Klinik und ihrer persönlichen Integrität können sie zur institutionellen Verankerung der Karriereförderung beitragen. Bei der Umsetzung der einzelnen Karriereschritte sind ihre Ideen gefragt und ihre fachliche Beratung notwendig. Ausserdem unterstützen sie die jüngeren Kolleginnen und Kollegen, wichtige Kontakte in der «scientific community» zu knüpfen. Auch die immer wieder neue Motivierung bei Rückschlägen oder Schwierigkeiten in der Umsetzung der Karrierepläne ist Aufgabe der Mentorinnen/Mentoren. Nicht zuletzt spielt das persönliche Vorbild eines Mentors/einer Mentorin eine wichtige Rolle.

- Die Mentorinnen/Mentoren sollten Gratifikationen erhalten durch individuelle und institutionelle Wertschätzung für ihre Mentoringaktivitäten und Anerkennung für ihre eigene Karriere [10]. Die Zeit und das Engagement, welche sie für das Mentorieren aufbringen, könnten in ähnlicher Weise wie eine Publikation als Leistungsnachweis gezählt werden.

- Die Mentees sollten Bereitschaft zur Offenheit, Eigenverantwortlichkeit, Zielorientierung und Handlungsbereitschaft mitbringen. Auch die persönliche Entwicklung ist ein wichtiger Teil im Mentoringprozess.

Der längerfristige Erfolg von Mentoring erfordert eine breitabgestützte Akzeptanz solcher Förderprogramme in den medizinischen Institutionen. Aus unserer Sicht ist es in der Medizin nicht sinnvoll, bei der Nachwuchsförderung auf der Ebene der Assistierenden geschlechterdifferente Gruppen zu bilden. Es besteht die Gefahr, dass durch die Geschlechtersegregation wechselseitige Vorurteile verstärkt und Rivalitätsgefühle geschürt werden, die wiederum indirekt Ausschlussmechanismen hervorrufen. Vielmehr sollen talentierte und engagierte Nachwuchskräfte in gemischtgeschlechtlichen Gruppen angeleitet werden, sich gegenseitig zu unterstützen und gemeinsam Strategien für den Aufbau einer beruflichen Karriere zu entwickeln.

\section{Dank}

Wir danken Dr. med. Christiane Roth, Spitaldirektorin des USZ, welche das Mentoringprogramm ideell und materiell seit Beginn nachhaltig unterstützt.

Prof. Dr. med. Claus Buddeberg danken wir für konzeptuelle und methodische Beratung bei der Durchführung des Mentoringprogramms. 